\title{
An assessment of peer-led teaching sessions for final-year medical students in a district general hospital in the UK
}

\author{
Authors: Avraneel Talapatra, Ha Phuong Do Le, Helen Craggs and Yasmeen Hayat
}

\section{Introduction}

A peer teaching programme was designed by foundation year one doctors (FY1s) at a district general hospital to help final-year medical students with preparation for their final-year examinations and for their life as doctors. The aim of the teaching programme was to provide a friendly and accessible learning experience for students, taught by recent medical graduates.

\section{Methods}

A programme of once-weekly OSCE (objective structured clinical examination)-style teaching sessions was organised over a 6-week period, outside normal working hours. FY1s designed original but realistic OSCE-style stations across a wide range of specialties. FY1 tutors were invited to facilitate these teaching sessions, which were attended by final-year medical students. Feedback was collected from all FY1s and medical students.

\section{Results and discussion}

We involved the majority of the FY1 cohort at the hospital in the teaching. This ensured that a wide breadth of experience was available. Each session consisted of three individual 20-minute stations. This enabled students to complete the required tasks and receive immediate feedback.

It has been shown in similar programmes that students appreciate teaching from those who have had recent experiences of medical school and understand the struggles they go through. The students in our programme particularly praised the relevance of the stations to their final exams and the quality of the feedback that was provided. The mean score across all sessions when asked about both domains was 4.96 out of $5(99.2 \%)$, on a scale where 1 is poor and 5 is excellent. The mean score for the level of organisation of the sessions was 4.88 out of $5(97.6 \%)$. Students were given advice on how to augment their clinical skills, including history taking and examination. The students praised the focus on novel scenarios such as SBAR (situation, background, assessment and recommendation) handover, patient prioritisation and dealing with acutely unwell patients. Students appreciated the open and supportive nature of these sessions and felt that they could ask relevant questions, as necessary.

Authors: Royal Bolton Hospital, Bolton, UK
Adams et al showed that peer teaching helps to hone the knowledge and skills of tutors. ${ }^{2}$ The tutors in our programme praised the opportunities to support and mentor final-year students. They enjoyed being able to collaborate with and learn from their peers who have experience in other specialties. They felt more confident in providing constructive feedback.

\section{Conclusion}

Peer teaching benefits not only the tutees, but also the tutors. The students praised the relevance of the scenarios to their exams and the personalised feedback that was provided. There was a focus on maintaining an open and supportive nature to the sessions. Newly qualified FY1s were able to share their valuable skills and experience from transitioning into working life to the final-year students. We will continue to provide peer-based teaching in this format in the forthcoming years.

\section{References}

1 Lockspeiser TM, O'Sullivan P, Teherani A, Muller J. Understanding the experience of being taught by peers: the value of social and cognitive congruence. Adv Health Sci Educ 2008;13:361-72.

2 Adams BS, Hernandez EJ, Tang TS. "Learning by teaching": a peerteaching model for diversity training in medical school. Teach Learn Med 2004;16:60-3. 